\title{
The magnetic power spectrum in Faraday rotation screens
}

\author{
T. A. Enßlin and C. Vogt \\ Max-Planck-Institut für Astrophysik, Karl-Schwarzschild-Str.1, Postfach 1317, 85741 Garching, Germany \\ Received 30 July 2002 / Accepted 7 February 2003

\begin{abstract}
The autocorrelation function and similarly the Fourier-power spectrum of a rotation measure (RM) map of an extended background radio source can be used to measure components of the magnetic autocorrelation and power-spectrum tensor within a foreground Faraday screen. It is possible to reconstruct the full non-helical part of this tensor in the case of an isotropic magnetic field distribution statistics. The helical part is only accessible with additional information; e.g. the knowledge that the fields are force-free. The magnetic field strength, energy spectrum and autocorrelation length $\lambda_{B}$ can be obtained from the non-helical part alone. We demonstrate that $\lambda_{B}$ can differ substantially from $\lambda_{R M}$, the observationally easily accessible autocorrelation length of an $R M$ map. In typical astrophysical situation $\lambda_{R M}>\lambda_{B}$. Any $R M$ study, which does not take this distinction into account, likely underestimates the magnetic field strength. For power-law magnetic power spectra, and for patchy magnetic field configurations the central $R M$ autocorrelation function is shown to have characteristic asymptotic shapes. Ways to constrain the volume filling factor of a patchy field distribution are discussed. We discuss strategies to analyse observational data, taking into account - with the help of a window function - the limited extent of the polarised radio source, the spatial distribution of the electron density and average magnetic energy density in the screen, and allowing for noise reducing data weighting. We briefly discuss the effects of possible observational artefacts, and strategies to avoid them.
\end{abstract}

Key words. magnetic fields - radiation mechanism: non-thermal - galaxies: active - intergalactic medium - galaxies: clusters: general - radio continuum: general

\section{Introduction}

\subsection{Cosmic magnetic fields}

The interstellar and intergalactic plasma is magnetised. The origin of the magnetic fields is partly a mystery, yet it allows fascinating insights into dynamical processes in the Universe. Magnetic fields are an important constituent of cosmic plasma in so far as they couple the often collisionless charged particles by the Lorentz-force. They are able to inhibit transport processes like heat conduction, spatial mixing of gas, and propagation of cosmic rays. They are essential for the acceleration of cosmic rays. They mediate forces through their tension and pressure, giving the plasma additional macroscopic degrees of freedom in terms of Alfvénic and magnetosonic waves. They allow distant cosmic ray electron populations to be observed by magneto-curvature (synchrotron) radiation.

Observational studies of spiral galaxies have revealed highly organised magnetic field configurations, often in alignment with the optical spiral arms. These magnetic fields are believed to be generated and shaped by the dynamo action of the differentially rotating galaxy disks from some initial weak seed fields.

The seed fields could have many origins, ranging from outflows from stars and active galactic nuclei over battery effects

Send offprint requests to: T. A. Enßlin, e-mail: ensslin@mpa-garching.mpg.de in shock waves, in ionisation fronts, and in neutral gas-plasma interactions, up to being primordially generated in high energy processes like phase transitions or inflation during the very early Universe.

In order to learn more about the magnetic field origin, less processed plasma has to be studied. There is the possibility that the magnetic fields outside galaxies, in galaxy clusters and - if existing - even in the wider intergalactic space carry more information on the field's origin. In clusters, magnetic fields with a much lower degree of ordering, compared to the organised fields in spiral galaxies, have been detected. However, they may be highly processed by turbulent gas flows driven by galaxy cluster mergers, which may mask their origin. Regardless, cluster magnetic fields are an interesting laboratory to study magneto-hydrodynamical (MHD) turbulence, and are of great importance to understand thermal and non-thermal phenomena in the intra-cluster medium.

Despite their obvious importance for many astrophysical questions, and despite many observational efforts to measure their properties, our knowledge of galactic and intergalactic magnetic fields is still poor. For an overview on the present observational and theoretical knowledge the excellent review articles by Rees (1987), Wielebinski \& Krause (1993), Kronberg (1994), Beck et al. (1996), Kulsrud (1999), Beck (2001), Grasso \& Rubinstein (2001), Carilli \& Taylor (2002), and Widrow (2002) should be consulted. 


\subsection{Faraday rotation}

One way to probe magnetic fields is to use the Faraday rotation effect. Linearly polarised radio emission experiences a rotation of the polarisation plane when it transverses a plasma with a non-zero magnetic field component along its propagation direction. If the Faraday active medium is external to the source, a wavelength-square dependence of the polarisation angle measured can be observed and used to obtain the $R M$, which is the proportionality constant of this dependence. Such situations are realised in nature in cases where a polarised radio galaxy is located behind the magnetised medium of a galaxy, or behind or embedded in a galaxy cluster.

The focus of this work is on the analysis of $R M$ maps of Faraday screens, in which the fields are statistically isotropically distributed. This should be approximately fulfilled in galaxy clusters, but not in the highly organised spiral galaxies. However, our analysis should also give some insight into the statistics of $R M$ maps of galaxies, since many of the results do not strictly require perfect isotropy.

Magnetic fields in galaxy clusters are known to exist due to detection of cluster wide synchrotron emission (Willson 1970), and detection of their Faraday rotation effect. Although the association of the $R M$ with the intra-cluster medium is not unambiguous, since it could also be produced in a magnetised plasma skin of the observed radio galaxy (Bicknell et al. 1990), there are arguments in favour of such an interpretation: (i) the asymmetric depolarisation of double radio lobes embedded in galaxy clusters can be understood as resulting from a difference in the Faraday depth of the two lobes (Laing 1988; Garrington et al. 1988). (ii) A recent $R M$ study by Clarke et al. (2001) of point sources located mostly behind (but $40 \%$ inside) galaxy clusters show a larger dispersion in $R M$ values than a reference sample without a galaxy cluster intersecting the line-of-sight. (iii) The cluster-wide radio halos observed in some clusters of galaxies (e.g. Feretti 1999) show synchrotron emission of relativistic electrons within magnetic fields. The cluster fields strength should be within an order of magnitude of their Faraday rotation estimates for the radio emitting electrons to have a reasonable energy density (compared to the thermal one) (e.g. as can be read off Fig. 1 in Enßlin \& Biermann 1998).

Typical $R M$ values of galaxy clusters are of the order of a few $100 \mathrm{rad} / \mathrm{m}^{2}$, being consistent with field strengths of a few $\mu \mathrm{G}$ which are well below equipartition with the thermal cluster gas. However, in cooling flow clusters extreme $R M$ values of a few $1000 \mathrm{rad} / \mathrm{m}^{2}$ were detected (see Carilli \& Taylor 2002), indicating possibly substantial magnetic pressure support of the intra-cluster gas there.

Although the magnetic fields of galaxy clusters are less ordered than these of spiral galaxies, the presence of coherent structures is suggested by high resolution Faraday maps, which exhibit sometimes $R M$ bands (e.g. Dreher et al. 1987; Taylor \& Perley 1993; Taylor et al. 2001; Eilek \& Owen 2002). Such bands may be caused by shear-amplification of originally small-scale magnetic fields, as seen in numerical MHD simulations of galaxy cluster formation (Dolag et al. 1999). They are likely embedded within a magnetic power-spectrum which extends over several orders of magnitude in wavevector space.
Similar to hydrodynamical turbulence, a broad energy injection range is followed by a power-law spectrum at larger wavevectors. For attempts to measure the magnetic power spectrum from cluster simulations and radio maps see Dolag et al. (2002) and Govoni et al. (2002) respectively ${ }^{1}$.

Another area of application of the theory developed here can be to measure the properties of an hypothetical large-scale magnetic field outside clusters of galaxies, which could be of primordial origin. A pioneering feasibility study in this direction was done by Kolatt (1998), who already outlined several of the ideas investigated in this work. He proposed to probe the cosmological magnetic fields by using catalogues of $R M$ measurements of distant radio galaxies and to measure spatial $R M$ correlations between them in order to measure the magnetic power spectrum, as we propose to do for extended Faraday rotation maps. An RM search for fields in the Lyman$\alpha$ forest by Oren \& Wolfe (1995) found at most a marginal detection. If they exist, primordial magnetic fields may be detectable by Faraday rotation of the cosmic microwave background (CMB) polarisation during and shortly after the epoch of recombination, as Kosowsky \& Loeb (1996) proposed.

Ohno et al. (2002) proposed to use the CMB polarisation even for $R M$ studies of nearby galaxy clusters. Should this speculative proposal become technically feasible, a lot of detailed information on intra-cluster magnetic fields could be obtained.

\subsection{Philosophy of the paper}

If magnetic fields are sampled in a sufficiently large volume, they can hopefully be regarded to be statistically homogeneous and statistically isotropic. This means that any statistical average of a quantity depending on the magnetic fields does not depend on the exact location, shape, orientation and size of the used sampling volume.

The quantity we are interested in this paper is the autocorrelation (or two-point-correlation) function (more exactly: tensor) of the magnetic fields. The information contained in the autocorrelation function is equivalent to the information stored in the power-spectrum, as stated by the Wiener-Khinchin Theorem (WKT). We therefore present two equivalent approaches, one based in real space, and one based in Fourier space. The advantage of this redundancy is that some quantities are easier accessible in one, and others in the other space. Further, this allows to crosscheck computer algorithms based on this work by comparing results gained by the different approaches.

The observable we can use to access the magnetic fields is Faraday rotation maps of extended polarised radio sources located behind a Faraday screen. Since an $R M$ map shows basically the line-of-sight projected magnetic field distribution, the $R M$ autocorrelation function is mainly given by the projected magnetic field autocorrelation function. Therefore measuring the $R M$ autocorrelation allows to measure the magnetic

\footnotetext{
1 The conventions to describe the spectra may differ in these articles from the one used here.
} 
autocorrelation, and thus provides a tool to estimate magnetic field strength and correlation length.

The situation is a bit more complicated than described above, due to the vector nature of the magnetic fields. This implies that there is an autocorrelation tensor instead of a function, which contains nine numbers corresponding to the correlations of the different magnetic components against each other, which in general can all be different. The $R M$ autocorrelation function contains only information about one of these values, the autocorrelations of the magnetic field component parallel to the line-of-sight. However, in many instances the important symmetric part of the tensor can be reconstructed and using this information the magnetic field strength and correlation length can be obtained. This is possible due to three observations:

1. Magnetic isotropy: if the sampling volume is sufficiently large, so that the local anisotropic nature of magnetic field distributions is averaged out, the (volume averaged) magnetic autocorrelation tensor is isotropic. This means, that the diagonal elements of the tensor are all the same, and that the off-diagonal elements are described by two numbers, one giving their symmetric, and one giving their antisymmetric (helical) contribution.

2. Divergence-freeness of magnetic fields: the condition $\boldsymbol{\nabla} \cdot \boldsymbol{B}=0$ ( $\boldsymbol{B}$ is the magnetic field) couples the diagonal and off-diagonal components of the symmetric part of the autocorrelation tensor. Knowledge of one diagonal element (e.g. from an $R M$ measurement) therefore specifies fully the symmetric part of the tensor. The trace of the autocorrelation tensor, which can be called scalar magnetic autocorrelation function $w(r)$, contains all the information required to measure the average magnetic energy density $\varepsilon_{B}=w(0) /(8 \pi)$ or the magnetic correlation length $\lambda_{B}=\int_{-\infty}^{\infty} \mathrm{d} r w(r) / w(0)$.

3. Unimportance of helicity: although helicity is a crucial quantity for the dynamics of magnetic fields, it does not enter any estimate of the average magnetic energy density, or magnetic correlation length, because helicity only affects off-diagonal terms of the autocorrelation tensor. The named quantities depend only on the trace of the tensor and are therefore unaffected by helicity. One cannot measure helicity from a Faraday rotation map alone, since it requires the comparison of two different components of the magnetic fields, whereas the $R M$ map contains information on only one component.

In a realistic situation, the sampling volume is determined by the shape of the polarised radio emitter and the geometry of the Faraday screen, as given by the electron density and the magnetic field energy density profile. The sampling volume can be described by a window function, through which an underlying virtually statistical homogeneous magnetic field is observed. The window function is zero outside the probed volume, e.g. for locations which are not located in front of the radio source. Inside the volume the window function scales with the electron density (known from X-ray observations), with the average magnetic energy profile (guessed from reasonable scaling relations, but testable within the approach), and - if wanted - with a noise reducing data weighting scheme. The effect of a finite window function is to smear out the power in Fourierspace. Since this is an unwanted effect one either has to find systems which provide a sufficiently large window or one has to account for this bias. Since the effect of a too small window on the results depends strongly on the shape and size of the window at hand, a detailed discussion of all the cases which can happen in practice is beyond the scope of this paper. It has to be done for each application at hand separately. Only idealised cases are discussed here for illustration. But generally one can state, that the analysis is sensitive to magnetic power on scales below a typical window size, and insensitive to scales above.

The same magnetic power spectrum can have very different realizations, since all the phase information is lost in measuring the power spectrum (for an instructive visualisation of this see Maron \& Goldreich 2001). Since the presented approach relies on the power spectrum only, it is not important if the magnetic fields are highly organised in structures like flux-ropes, or magnetic sheets, or if they are relatively featureless random-phase fields, as long as their power spectrum is the same.

The autocorrelation analysis is fully applicable in all such situations, as long as the fields are sampled with sufficient statistics. The fact that this analysis is insensitive to different realizations of the same power spectrum indicates that the method is not able to extract all the information which may be in the map. Additional information is stored in higher order correlation functions, and such can in principle be used to make statements about whether the fields are ordered or purely random (chaotic). The information on the magnetic field strength $\left(\boldsymbol{B}^{2}\right.$, which is the value at origin of the autocorrelation function), and correlation length (an integral over the autocorrelation function) does only depend on the autocorrelation function and not on the higher order correlations.

The presented analysis relies on having a statistically isotropic sample of magnetic fields, whereas MHD turbulence seems to be locally inhomogeneous, which means that small scale fluctuations are anisotropic with respect to the local mean field. However, whenever the observing window is much larger than the correlation length of the local mean field the autocorrelation tensor should be isotropic due to averaging over an isotropic distribution of locally anisotropic subvolumes. This works if not a preferred direction is superposed by other physics, e.g. a galaxy cluster wide orientation of field lines along a preferred axis. However, even this case can in principle be treated by co-adding the $R M$ signal from a sample of clusters, for which a random distribution of such hypothetical axes can be assumed. In any case, it is likely that magnetic anisotropy also manifests itself in the Faraday rotation maps, since the projection connecting magnetic field configurations and $R M$ maps will conserve anisotropy in most cases, except alignments by chance of the direction of anisotropy and the line-of-sight. The presence of anisotropy can therefore be tested, which is discussed later in great detail.

Since there are cases where already an inspection by eye seems to reveal the existence of magnetic structures like flux ropes or magnetic sheets, we briefly discuss their appearance in the autocorrelation and the area filling statistics of $R M$ maps. 
As already stated, the presence of such structures does not limit our analysis, as long as they are sufficiently sampled. Otherwise, one has to replace e.g. the isotropy assumption by a suitable generalisation. In many cases this will allow an analysis similar to the one proposed in this paper. We leave this for future work and applications where this might be required. A criteria to detect anisotropy statistically is given in this work.

\subsection{Structure of the paper}

In Sect. 2 the autocorrelation functions of magnetic fields and their $R M$ maps are introduced, and their interrelation investigated. In Sect. 3 the same is done in Fourier space, which has not only technical advantages, but also provides insight into phenomena such as turbulence. Faraday map signatures of magnetic structures like flux-ropes are briefly discussed in Sect. 4. Possible pitfalls due to observational artefacts are investigated in Sect. 5. The conclusions in Sect. 6 summarise our main findings, and give references to the important results and formulae in detail.

\section{Real space formulation}

\subsection{Basics}

The Faraday rotation for a line-of-sight parallel to the $z$-axis and displaced by $\boldsymbol{x}_{\perp}$ from it, which starts at a polarised radio source at $z_{\mathrm{s}}\left(\boldsymbol{x}_{\perp}\right)$ and ends at the observer located at infinity is given by

$R M\left(\boldsymbol{x}_{\perp}\right)=a_{0} \int_{z_{\mathrm{s}}\left(\boldsymbol{x}_{\perp}\right)}^{\infty} \mathrm{d} z n_{\mathrm{e}}(\boldsymbol{x}) B_{z}(\boldsymbol{x})$,

where $a_{0}=e^{3} /\left(2 \pi m_{\mathrm{e}}^{2} c^{4}\right), \boldsymbol{x}=\left(\boldsymbol{x}_{\perp}, z\right), n_{\mathrm{e}}$ the electron density, and $\boldsymbol{B}$ the magnetic field strength. We assume in the following that any Faraday rotation due to a foreground as the Galaxy or the Earth's ionosphere is subtracted from the $R M$ values, and only the $R M$ of the Faraday screen is remaining. We also neglect any redshift effects, which can be included by inserting the factor $\left(1+z_{\text {redshift }}(z)\right)^{-2}$ into the integrand.

The focus of this work is on the statistical expectation of the two-point, or autocorrelation function of Faraday rotation maps. This is defined by

$C_{R M}\left(\boldsymbol{x}_{\perp}, \boldsymbol{r}_{\perp}\right)=\left\langle R M\left(\boldsymbol{x}_{\perp}\right) R M\left(\boldsymbol{x}_{\perp}+\boldsymbol{r}_{\perp}\right)\right\rangle$,

where the brackets indicate the expectation value of a statistical ensemble average, which in practice may be replaced by a suitable average, e.g. over an observed $R M$ map. For a given polarised background radio source of projected area $\Omega$ we define the observable correlation function as

$C_{R M}^{\mathrm{obs}}\left(\boldsymbol{r}_{\perp}\right)=\frac{1}{A_{\Omega}} \int \mathrm{d} x_{\perp}^{2} R M\left(\boldsymbol{x}_{\perp}\right) R M\left(\boldsymbol{x}_{\perp}+\boldsymbol{r}_{\perp}\right)$,

where it is assumed that $R M\left(x_{\perp}\right)=0$ for $\boldsymbol{x}_{\perp} \notin \Omega$. $A_{\Omega}$ is taken here to be the radio source area, although other normalisations are imaginable, e.g. one might choose the area over which $\boldsymbol{x}_{\perp}$ and $\boldsymbol{x}_{\perp}+\boldsymbol{r}_{\perp}$ are simultaneously within $\Omega$. This latter weighting would give a statistically unbiased estimator of $C_{R M}\left(\boldsymbol{r}_{\perp}\right)$, but we do not recommend its usage for the following reasons.
The resulting estimator has a strong sensitivity to poorly sampled variances on scales comparable to the radio source diameter. On larger scales it is also ill-defined in the mathematical sense. The normalisation proposed here leads to results which do not suffer from this. It automatically down-weights the signal from statistically insufficiently sampled baselines $\boldsymbol{r}_{\perp}$, and this bias can be controlled (Sect. 3.5). It further turns out that the Fourier-space formulation of $R M$ statistics introduced in Sect. 3 requires the weighting scheme proposed here.

The $R M$ signal from different subvolumes of the Faraday screen will differ due to electron density and typical magnetic field strength variations within the source. Such global variations can be regarded as variations of a window function $f(\boldsymbol{x})$, which mediates the relation between the observed $R M$ signal and an underlying (rescaled) magnetic field, which is virtually homogeneous in a statistical sense. To be more specific, we choose a typical position $\boldsymbol{x}_{\text {ref }}$ within the screen (e.g. the centre of a galaxy cluster), and define $n_{\mathrm{e} 0}=n_{\mathrm{e}}\left(\boldsymbol{x}_{\mathrm{ref}}\right)$ and $B_{0}=\left\langle B^{2}\left(\boldsymbol{x}_{\text {ref }}\right)\right\rangle^{1 / 2}$. We then define the window function by

$f(\boldsymbol{x})=\mathbf{1}_{\left\{\boldsymbol{x}_{\perp} \in \Omega\right\}} \mathbf{1}_{\left\{z \geq z_{\mathrm{s}}\left(\boldsymbol{x}_{\perp}\right)\right\}} h\left(\boldsymbol{x}_{\perp}\right) g(\boldsymbol{x}) n_{\mathrm{e}}(\boldsymbol{x}) / n_{\mathrm{e} 0}$,

where $\mathbf{1}_{\{\text {condition\} }}$ is defined to be 1 if condition is true, otherwise 0. $g(\boldsymbol{x})=\left\langle B^{2}(\boldsymbol{x})\right\rangle^{1 / 2} / B_{0}$ is the dimensionless average magnetic field profile. In galaxy clusters a reasonable working assumption $^{2}$ for this may be $g(\boldsymbol{x})=\left(n_{\mathrm{e}}(\boldsymbol{x}) / n_{\mathrm{e} 0}\right)^{\alpha_{B}}$, for which we expect $\alpha_{B} \approx 1$ (e.g. see Dolag et al. 2001). The function $h\left(\boldsymbol{x}_{\perp}\right)$ allows us to assign different pixels in the map different weights ${ }^{3}$, e.g. in cases where the noise is a function of the position one might want to down-weight noisy regions ${ }^{4}$. If no weighting applies $h\left(\boldsymbol{x}_{\perp}\right)=1$ everywhere.

The expectation of the observed $R M$ correlations are

$$
\begin{aligned}
\left\langle C_{R M}^{\mathrm{obs}}\left(\boldsymbol{r}_{\perp}\right)\right\rangle= & \frac{a_{0}^{2} n_{\mathrm{e} 0}^{2}}{A_{\Omega}} \int \mathrm{d}^{3} x \int_{-\infty}^{\infty} \mathrm{d} r_{z} \\
& \times f(\boldsymbol{x}) f(\boldsymbol{x}+\boldsymbol{r})\left\langle\tilde{B}_{z}(\boldsymbol{x}) \tilde{B}_{z}(\boldsymbol{x}+\boldsymbol{r})\right\rangle,
\end{aligned}
$$

with $\boldsymbol{r}=\left(\boldsymbol{r}_{\perp}, r_{z}\right)$, and $\tilde{\boldsymbol{B}}(\boldsymbol{x})=\boldsymbol{B}(\boldsymbol{x}) / g(\boldsymbol{x})$ the rescaled magnetic field. If properly rescaled, the average strength of the field is independent of the position. In that case, the rescaled

\footnotetext{
${ }^{2}$ We note that the average magnetic field strength profile can be tested a posteriori for consistency with the data, by comparing the observed values of $R M^{2}\left(x_{\perp}\right)$ to the model expectations (see Sect. 2.4).

${ }^{3}$ If $h\left(x_{\perp}\right) \neq 1$ for some $x_{\perp} \in \Omega$ the corresponding weight has to be introduced into the analysis. The most efficient way to do this is to make the data weighting virtually part of the measurement process, by writing $R M\left(x_{\perp}\right) \rightarrow R M^{\mathrm{w}}\left(\boldsymbol{x}_{\perp}\right)=R M\left(\boldsymbol{x}_{\perp}\right) h\left(\boldsymbol{x}_{\perp}\right)$. For convenience, we drop the superscript ${ }^{\mathrm{W}}$ again in the following, and just note that the analysis described here has to be applied to the weighted data $R M^{\mathrm{w}}\left(\boldsymbol{x}_{\perp}\right)$.

${ }^{4}$ If $\sigma\left(x_{\perp}\right)$ is the noise of the $R M$ map at position $x_{\perp}$ a reasonable choice of a weighting function would be $h\left(\boldsymbol{x}_{\perp}\right)=\sigma_{0} / \sigma\left(\boldsymbol{x}_{\perp}\right)$. If the noise map itself could have errors, the danger of over-weighting noisy pixels with underestimated noise can be avoided by thresholding: if $\sigma_{0}$ is a threshold below which the noise is regarded to be tolerable, we propose to use $h\left(\boldsymbol{x}_{\perp}\right)=1 /\left(1+\sigma\left(\boldsymbol{x}_{\perp}\right) / \sigma_{0}\right)$. Another choice would be $h\left(\boldsymbol{x}_{\perp}\right)=\mathbf{1}_{\left\{\sigma\left(x_{\perp}\right)<\sigma_{0}\right\}}$ which just cuts out regions which are recognised as too noisy.
} 
magnetic field autocorrelation tensor should also be independent of position:

$M_{i j}(\boldsymbol{r})=\left\langle\tilde{B}_{i}(\boldsymbol{x}) \tilde{B}_{j}(\boldsymbol{x}+\boldsymbol{r})\right\rangle$.

If the spatial variation of the window function is on much larger scales than the correlation length $\lambda_{B}$ of the magnetic fields, then Eq. (5) can be approximated to be

$$
\begin{aligned}
& C_{R M}\left(\boldsymbol{r}_{\perp}\right)=\left\langle C_{R M}^{\mathrm{obs}}\left(\boldsymbol{r}_{\perp}\right)\right\rangle=a_{1} C_{\perp}\left(\boldsymbol{r}_{\perp}\right), \text { with } a_{1}=a_{0}^{2} n_{\mathrm{e} 0}^{2} L, \\
& C_{\perp}\left(\boldsymbol{r}_{\perp}\right)=\int_{-\infty}^{\infty} \mathrm{d} r_{z} M_{z z}(\boldsymbol{r}), \text { and } \boldsymbol{r}=\left(\boldsymbol{r}_{\perp}, r_{z}\right) .
\end{aligned}
$$

Here, we introduced the characteristic depth of the Faraday screen $L=V_{[f]} / A_{\Omega}$, where $V_{[f]}=\int \mathrm{d} x^{3} f^{2}(\boldsymbol{x})$ is the probed effective volume. We also introduced for convenience the normalised $R M$ autocorrelation function $C_{\perp}$ which differs from $C_{R M}$ only by a geometry dependent factor $a_{1}$.

In the following we ignore the influence of the window function in the discussion, since for sufficiently large windows it only affects $a_{1}$. We therefore write $\boldsymbol{B}$ for $\tilde{\boldsymbol{B}}$ and keep in mind that our measured field strength $B_{0}$ is estimated for a volume close to the reference location $\boldsymbol{x}_{\text {ref }}$. At other locations, the average magnetic energy density is given by $\varepsilon_{B}(\boldsymbol{x})=g^{2}(\boldsymbol{x}) B_{0}^{2}$. This approach assumes implicitly that typical length scales are the same throughout the Faraday screen. For sufficiently extended screens, this assumption can be tested by comparing results from different and separately analysed regions of the $R M$ map.

\subsection{Isotropic magnetic correlation tensor}

The magnetic autocorrelation tensor for homogeneous isotropic turbulence, as assumed throughout the rest of this paper, can be written as

$M_{i j}(\boldsymbol{r})=M_{\mathrm{N}}(r) \delta_{i j}+\left(M_{\mathrm{L}}(r)-M_{\mathrm{N}}(r)\right) \frac{r_{i} r_{j}}{r^{2}}+M_{\mathrm{H}}(r) \epsilon_{i j k} r_{k}$

(e.g. Subramanian 1999) where the longitudinal, normal, and helical autocorrelation functions, $M_{\mathrm{L}}(r), M_{\mathrm{N}}(r)$, and $M_{\mathrm{H}}(r)$ respectively, only depend on the distance, not on the direction. The condition $\boldsymbol{\nabla} \cdot \boldsymbol{B}=0$ leads to $\partial / \partial r_{i} M_{i j}(\boldsymbol{r})=0$ (here and below we make use of the sum convention). This allows us to connect the non-helical correlation functions by

$M_{\mathrm{N}}(r)=\frac{1}{2 r} \frac{\mathrm{d}}{\mathrm{d} r}\left(r^{2} M_{\mathrm{L}}(r)\right)$

(Subramanian 1999). The $z z$-component of the magnetic autocorrelation tensor depends only on the longitudinal and normal correlations, and not on the helical part:

$M_{z z}(\boldsymbol{r})=M_{\mathrm{L}}(r) \frac{r_{z}^{2}}{r^{2}}+M_{\mathrm{N}}(r) \frac{r_{\perp}^{2}}{r^{2}}$ with $\boldsymbol{r}=\left(\boldsymbol{r}_{\perp}, r_{z}\right)$,

which implies that Faraday rotation is insensitive to magnetic helicity. It is also useful to introduce the magnetic autocorrelation function

$w(\boldsymbol{r})=\langle\boldsymbol{B}(\boldsymbol{x}) \cdot \boldsymbol{B}(\boldsymbol{x}+\boldsymbol{r})\rangle=M_{i i}(\boldsymbol{r})$,

which is the trace of the autocorrelation tensor, and depends only on $r$ (in the case of a statistically isotropic magnetic field distribution, in the following called briefly isotropic turbulence):

$w(\boldsymbol{r})=w(r)=2 M_{\mathrm{N}}(r)+M_{\mathrm{L}}(r)=\frac{1}{r^{2}} \frac{\mathrm{d}}{\mathrm{d} r}\left(r^{3} M_{\mathrm{L}}(r)\right)$.

In the last step Eq. (9) was used. Since the average magnetic energy density is given by $\left\langle\varepsilon_{B}\right\rangle=w(0) /(8 \pi)$ the magnetic field strength can be determined by measuring the zero-point of $w(r)$. This can be done by Faraday rotation measurements: The $R M$ autocorrelation can be written as

$$
C_{\perp}\left(r_{\perp}\right)=\frac{1}{2} \int_{-\infty}^{\infty} \mathrm{d} r_{z} w\left(\sqrt{r_{\perp}^{2}+r_{z}^{2}}\right)=\int_{r_{\perp}}^{\infty} \mathrm{d} r \frac{r w(r)}{\sqrt{r^{2}-r_{\perp}^{2}}} .
$$

and is therefore just a line-of-sight projection of the magnetic autocorrelation. Thus the magnetic autocorrelations $w(r)$ can be derived from $C_{\perp}\left(r_{\perp}\right)$ by inverting an Abel integral equation:

$$
\begin{aligned}
w(r) & =-\frac{2}{\pi r} \frac{\mathrm{d}}{\mathrm{d} r} \int_{r}^{\infty} \mathrm{d} y \frac{y C_{\perp}(y)}{\sqrt{y^{2}-r^{2}}} \\
& =-\frac{2}{\pi} \int_{r}^{\infty} \mathrm{d} y \frac{C_{\perp}^{\prime}(y)}{\sqrt{y^{2}-r^{2}}}
\end{aligned}
$$

where the prime denotes a derivative. For the second equation it was used that $w(r)$ stays bounded for $r \rightarrow \infty$.

Now, an observational program to measure magnetic fields is obvious: from a high quality Faraday rotation map of a homogeneous, (hopefully) isotropic medium of known geometry and electron density (e.g. derived from $\mathrm{X}$-ray maps) the $R M$ autocorrelation has to be calculated (Eq. (7)). From this an Abel integration (Eqs. (14) or (15)) leads to the magnetic autocorrelation function, which gives $\left\langle B^{2}\right\rangle$ at its origin. Formally,

$\left\langle B^{2}\right\rangle=w(0)=-\frac{2}{\pi} \int_{0}^{\infty} \mathrm{d} y \frac{C_{\perp}^{\prime}(y)}{y}$,

but this formulation is notoriously sensitive to noise. More stable methods are presented later.

\subsection{Correlation volume, area, and length}

For isotropic magnetic turbulence statements about integrals of $w(r)$ and $C_{\perp}\left(r_{\perp}\right)$ can be made. If correlations are short ranged, in the sense that $M_{\mathrm{L}}(r) r^{3} \rightarrow 0$ for $r \rightarrow \infty$, e.g. because of a finite size of the magnetised volume, then Eq. (12) implies

$V_{B}=\frac{4 \pi}{w(0)} \int_{0}^{\infty} \mathrm{d} r r^{2} w(r)=0$.

This means that the magnetic autocorrelation volume $V_{B}$ is zero and $w(r)$ must therefore have positive and negative values if the magnetic field is non-zero. With the help of Eqs. (13) or (15) this can be translated into a statement on the more directly measurable $R M$ autocorrelation:

$A_{R M}=\frac{2 \pi}{C_{\perp}(0)} \int_{0}^{\infty} \mathrm{d} r_{\perp} r_{\perp} C_{\perp}\left(r_{\perp}\right)=0$.

Also $C_{\perp}(r)$ must have positive and negative values for a nonzero magnetic field, since the autocorrelation area $A_{R M}$ of the 
$R M$ map is zero. We note, that Eq. (18) can conveniently be expressed as

$A_{R M}=\frac{\left(\int \mathrm{d} x_{\perp} R M\left(x_{\perp}\right)\right)^{2}}{\int \mathrm{d} x_{\perp} R M\left(x_{\perp}\right)^{2}}$,

a form which should make clear that any foreground $R M$ has to be removed before this quantity can satisfy Eq. (18). Equations (17)-(19) are a direct consequence of $\boldsymbol{\nabla} \cdot \boldsymbol{B}=0$. These equations can be used to test how much a given observation (or model) deviates from giving a proper average of an isotropic ensemble.

For example, the frequently used magnetic cell-model, in which cells of length-scale $l_{\text {cell }}$ are filled with a from cell-to-cell randomly oriented but internally homogeneous magnetic field, does not fulfil $\boldsymbol{\nabla} \cdot \boldsymbol{B}=0$. As a consequence it gives positive autocorrelation volumes and surfaces of the order $V_{B} \sim l_{\text {cell }}^{3}$ and $A_{R M} \sim l_{\text {cell }}^{2}$, respectively. Therefore it should be possible to exclude such an oversimplified model observationally.

Other integral quantities of the turbulent magnetic field to look at are the existing non-zero magnetic and $R M$ autocorrelation lengths. The magnetic autocorrelation length can be defined as

$\lambda_{B}=\int_{-\infty}^{\infty} \mathrm{d} r \frac{w(r)}{w(0)}=2 \frac{C_{\perp}(0)}{w(0)}$

where for the derivation of the last expression Eqs. (13) or (15) can be used. Even in globally homogeneous turbulence, there is always a preferred direction defined by the local magnetic field. One can ask for the correlation length along and perpendicular to this locally defined direction and gets $\lambda_{\|}=\frac{3}{2} \lambda_{B}$, and $\lambda_{\perp}=$ $\frac{3}{4} \lambda_{B}$ so that $\lambda_{B}=\frac{1}{3}\left(\lambda_{\|}+2 \lambda_{\perp}\right){ }^{5}$

An observationally easily accessible length scale is the Faraday rotation autocorrelation length:

$\lambda_{R M}=\int_{-\infty}^{\infty} \mathrm{d} r_{\perp} \frac{C_{\perp}\left(r_{\perp}\right)}{C_{\perp}(0)}=\pi \frac{\int_{-\infty}^{\infty} \mathrm{d} r r w(r)}{\int_{-\infty}^{\infty} \mathrm{d} r w(r)}$.

From comparing Eqs. (20) and (21) it is obvious that the $R M$ correlation length-scale is not identical to the magnetic

\footnotetext{
5 The ratio of $\lambda_{\|} / \lambda_{\perp}=2$ seems to be small given the highly anisotropic nature of MHD turbulence (some recent progress on this can be found in Sridhar \& Goldreich 1994; Goldreich \& Sridhar 1997; Maron \& Goldreich 2001; Cho et al. 2002). But it has to be noted, that there usually the magnetic fluctuations on top of a mean field are investigated, whereas here the correlation lengths of an isotropically oriented magnetic field distribution sampled on length-scales above the scale of the locally present mean field of these studies, is investigated. The ratio $\lambda_{\|} / \lambda_{\perp}=2$ seems also to be in contradiction to the typical MHD turbulence picture of long and thin magnetic flux ropes with large aspect ratios. However, this is not necessarily the case. For a given position $\boldsymbol{x}_{\text {rope }}$ on a flux rope, the correlation length along and perpendicular will typically have a much larger ratio than $\lambda_{\|} / \lambda_{\perp}=2$. But the position $\boldsymbol{x}_{\text {rope }}$ is a special position, since it was selected to be a high field strength region, whereas the correlation length $\lambda_{\|}$and $\lambda_{\perp}$ are defined by the statistical average over all positions in the volume. In order to make statements about the presence or absence of magnetic filaments higher order statistics, beyond the two-point level used mostly in this work, have to be applied.
}

field autocorrelation length. As shown later, the $R M$ correlation length is more strongly weighted towards the largest lengthscales in the magnetic fluctuation spectrum than the magnetic correlation length. This is crucial, since in some cases these scales have been assumed to be identical, which could have led to systematic underestimates of magnetic field strengths.

Having now defined two characteristic length-scales of the fields and their RM maps, suitable criteria testing the observed autocorrelation volume and area for statistical completeness can be formulated: $\left|V_{B}^{\mathrm{obs}}\right| \ll \lambda_{B}^{3}$ and $\left|A_{R M}^{\mathrm{obs}}\right| \ll \lambda_{R M}^{2}$. We note that for strictly positive and therefore unphysical autocorrelation functions one would expect $\left|V_{B}^{\mathrm{obs}}\right| \sim \lambda_{B}^{3}$ and $\left|A_{R M}^{\mathrm{obs}}\right| \sim \lambda_{R M}^{2}$ statistically.

This can be turned around. For a sufficiently large $R M$ map of a Faraday rotation screen with isotropic magnetic fields any homogeneous $R M$ contribution from additional magnetised foregrounds can relatively accurately be measured. If there is a weak screen-intrinsic homogeneous magnetic field component, its $z$-component is

$$
\left\langle B_{z}\right\rangle=\frac{\int \mathrm{d}^{2} x_{\perp} R M\left(x_{\perp}\right)}{a_{0} n_{\mathrm{e} 0} \int \mathrm{d}^{3} x f\left(\boldsymbol{x}_{\perp}\right)},
$$

where in $f(\boldsymbol{x})$ the average magnetic field profile may be set to $g(\boldsymbol{x})=1$ everywhere for a truly homogeneous component.

\subsection{Testing the window function}

Now, all the necessary tools are introduced to test if the window function $f(\boldsymbol{x})$ was based on a sensible model for the average magnetic energy density profile $g^{2}(\boldsymbol{x})$ and the proper geometry of the radio source within the Faraday screen $z_{\mathrm{s}}\left(\boldsymbol{x}_{\perp}\right)$ (see Eq. (4)). Models can eventually be excluded a-posteriori on the basis of

$\chi^{2}\left(x_{\perp}\right)=\frac{R M\left(x_{\perp}\right)^{2}}{\left\langle R M\left(x_{\perp}\right)^{2}\right\rangle}$,

where for the expected $R M$ dispersion

$\left\langle R M\left(\boldsymbol{x}_{\perp}\right)^{2}\right\rangle=\frac{1}{2} a_{0}^{2} n_{0}^{2} B_{0}^{2} \lambda_{B} \int_{-\infty}^{\infty} \mathrm{d} z f^{2}(\boldsymbol{x})$

has to be used. As shown before $B_{0}$ (e.g. by Eq. (16)), and $\lambda_{B}$ (e.g. by Eq. (20)) can be derived for a given window function $f(\boldsymbol{x})$ using $C_{R M}^{\mathrm{obs}}\left(\boldsymbol{r}_{\perp}\right)$. For a good choice of the window function, one gets

$\chi_{\mathrm{av}}^{2}=\frac{1}{A_{\Omega}} \int \mathrm{d} x_{\perp}^{2} \chi^{2}\left(\boldsymbol{x}_{\perp}\right) \approx 1$,

and larger values if the true and assumed models differ significantly. The model discriminating power lies also in the spatial distribution $\chi^{2}\left(x_{\perp}\right)$, and not only in its global average. If some large scale trends are apparent, e.g. that $\chi^{2}\left(x_{\perp}\right)$ is systematically higher in more central or more peripheral regions of the Faraday screen, then such a model for $f(\boldsymbol{x})$ should be disfavoured. This can be tested by averaging $\chi^{2}\left(x_{\perp}\right)$ e.g. in radial bins for a roughly spherical screen, as a relaxed galaxy cluster should be, and checking for apparent trends. 
We note, that this method of model testing can be regarded as a refined Laing-Garrington effect (Laing 1988; Garrington et al. 1988): the more distant radio cocoon of a radio galaxy in a galaxy cluster is usually more depolarised than the nearer radio cocoon due to the statistically larger Faraday depth. This is observed whenever the observational resolution is not able to resolve the $R M$ structures. Here, we assume that the observational resolution is sufficient to resolve the $R M$ structures, so that a different depth of some part of the radio source observed, or a different average magnetic energy profile leads to a different statistical Faraday depth $\left\langle R M\left(x_{\perp}\right)^{2}\right\rangle$. Since this can be tested by suitable statistics, e.g. the simple $\chi^{2}$ statistic proposed here, incorrect models can be identified.

It may be hard in an individual case to disentangle the effect of changing the total depth $z_{\mathrm{s}}$ of the used polarised radio source if it is embedded in the Faraday screen, and the effect of changing $B_{0}^{\text {obs }}$, since these two parameters can be quite degenerate. However, there may be situations in which the geometry is sufficiently constrained because of additional knowledge of the source position, or statistical arguments can be used if a sufficiently large sample of similar systems were observed.

\section{Fourier space formulation}

\subsection{Basics}

We use the following convention for the Fourier transformation of a $n$-dimensional function $F(\boldsymbol{x})$ :

$$
\begin{aligned}
& \hat{F}(\boldsymbol{k})=\int \mathrm{d}^{n} x F(\boldsymbol{x}) \mathrm{e}^{i \boldsymbol{k} \cdot \boldsymbol{x}} \\
& F(\boldsymbol{x})=\frac{1}{(2 \pi)^{n}} \int \mathrm{d}^{n} k \hat{F}(\boldsymbol{k}) \mathrm{e}^{-i \boldsymbol{k} \cdot \boldsymbol{x}} .
\end{aligned}
$$

The Fourier transformed isotropic magnetic autocorrelation tensor reads

$\hat{M}_{i j}(\boldsymbol{k})=\hat{M}_{\mathrm{N}}(k)\left(\delta_{i j}-\frac{k_{i} k_{j}}{k^{2}}\right)-i \varepsilon_{i j m} \frac{k_{m}}{k} \hat{H}(k)$,

where we have directly used the $\boldsymbol{\nabla} \cdot \boldsymbol{B}=0$ condition in the form $k_{i} \hat{M}_{i j}(\boldsymbol{k})=0$ to reduce the degrees of freedom to two components, a normal and helical part. The two corresponding spherically symmetric functions in $k$-space are given in terms of their real space counterparts as:

$$
\begin{aligned}
\hat{M}_{\mathrm{N}}(k) & =\int \mathrm{d}^{3} r M_{\mathrm{N}}(r) \mathrm{e}^{i k \cdot r}=4 \pi \int_{0}^{\infty} \mathrm{d} r r^{2} M_{\mathrm{N}}(r) \frac{\sin (k r)}{k r} \\
\hat{H}(k) & =\frac{\mathrm{d}}{\mathrm{d} k} \hat{M}_{\mathrm{H}}(k)=\frac{\mathrm{d}}{\mathrm{d} k} \int \mathrm{d}^{3} r M_{\mathrm{H}}(r) \mathrm{e}^{i k \cdot r}, \\
& =\frac{4 \pi}{k} \int_{0}^{\infty} \mathrm{d} r r^{2} M_{\mathrm{H}}(r) \frac{k r \cos (k r)-\sin (k r)}{k r} .
\end{aligned}
$$

One can also introduce the Fourier transformed trace of the autocorrelation tensor $\hat{w}(\boldsymbol{k})=\hat{M}_{i i}(\boldsymbol{k})=2 \hat{M}_{\mathrm{N}}(k)$. A comparison with the transformed $z z$-component of the autocorrelation tensor

$$
\hat{M}_{z z}(\boldsymbol{k})=\hat{M}_{\mathrm{N}}(k)\left(1-k_{z}^{2} / k^{2}\right)
$$

reveals that in the $k_{z}=0$ plane these two functions are identical (up to a constant factor 2). Since the 2-d Fourier transformed normalised $R M$ map is also identical to this, as a transformation of Eq. (7) shows, we can state

$$
\hat{C}_{\perp}\left(\boldsymbol{k}_{\perp}\right)=\hat{M}_{z z}\left(\boldsymbol{k}_{\perp}, 0\right)=\frac{1}{2} \hat{w}\left(\boldsymbol{k}_{\perp}, 0\right) .
$$

This Fourier-space version of Eq. (13) says, that the 2-d transformed $R M$ map reveals the $k_{z}=0$ plane of $\hat{M}_{z z}(\boldsymbol{k})$, which in the isotropic case is all what is required to reconstruct the full magnetic autocorrelation $\hat{w}(k)=2 \hat{C}_{\perp}(k)$.

\subsection{Power spectra}

We recall that the power spectrum $P_{[F]}(\boldsymbol{k})$ of a function $F(\boldsymbol{x})$ is given by the absolute-square of its Fourier transformation $P_{[F]}(\boldsymbol{k})=|\hat{F}(\boldsymbol{k})|^{2}$. The WKT states that the Fourier transformation of an autocorrelation function $C_{[F]}(\boldsymbol{r})$, estimated within a window with volume $V_{n}$ (as in Eq. (3)), gives the (windowed) power spectrum of this function, and vice versa:

$P_{[F]}(\boldsymbol{k})=V_{n} \hat{C}_{[F]}(\boldsymbol{k})$.

The WKT allows us to write the Fourier transformed autocorrelation tensor as

$\hat{M}_{i j}(\boldsymbol{k})=\frac{1}{V}\left\langle\hat{B}_{i}(\boldsymbol{k}) \overline{\hat{B}_{j}(\boldsymbol{k})}\right\rangle$,

where $V$ denotes the volume of the window function, which is for practical work with $R M$ maps often the probed effective volume $V=V_{[f]}$ as defined in Sect. 2.1.

Thus, the 3-d magnetic power spectrum (the Fourier transformed magnetic autocorrelation function $w(\boldsymbol{r})$ ) can be directly connected to the one-dimensional magnetic energy spectrum in the case of isotropic turbulence:

$\varepsilon_{B}(k) \mathrm{d} k=\frac{4 \pi k^{2}}{(2 \pi)^{3}} \frac{\hat{w}(k)}{8 \pi} \mathrm{d} k=\frac{k^{2} \hat{w}(k)}{2(2 \pi)^{3}} \mathrm{~d} k$,

where we wrote $\hat{w}(\boldsymbol{k})=\hat{w}(k)$ due to isotropy. The WKT also connects the 2-dimensional Fourier-transformed $R M$ map with the Fourier transformed $R M$ autocorrelation function:

$\hat{C}_{\perp}\left(k_{\perp}\right)=\frac{\left\langle\left|R \hat{M}\left(k_{\perp}\right)\right|^{2}\right\rangle}{a_{1} A_{\Omega}}$.

Thus, by comparing Eqs. (32), (35), and (36) one finds that the magnetic energy spectrum is most easily measured from a given observation by simply Fourier transforming the map $R M\left(x_{\perp}\right)$, and averaging this over rings in $\boldsymbol{k}_{\perp}$-space:

$\varepsilon_{B}^{\mathrm{obs}}(k)=\frac{k^{2}}{a_{1} A_{\Omega}(2 \pi)^{4}} \int_{0}^{2 \pi} \mathrm{d} \phi\left|R \hat{M}\left(\boldsymbol{k}_{\perp}\right)\right|^{2}$,

where $\boldsymbol{k}_{\perp}=k(\cos \phi, \sin \phi)$. Equation (37) gives a direct model independent observational route to measure the turbulent energy spectrum. The average magnetic energy density can be easily obtained from this via

$\varepsilon_{B}^{\mathrm{obs}}=\int_{0}^{\infty} \mathrm{d} k \varepsilon_{B}^{\mathrm{obs}}(k)=\int \mathrm{d}^{2} k_{\perp} \frac{k_{\perp}\left|R \hat{M}\left(\boldsymbol{k}_{\perp}\right)\right|^{2}}{a_{1} A_{\Omega}(2 \pi)^{4}}$,

where the last integration extends over the Fourier transformed $R M$ map and can be done in practice by summing over pixels. 

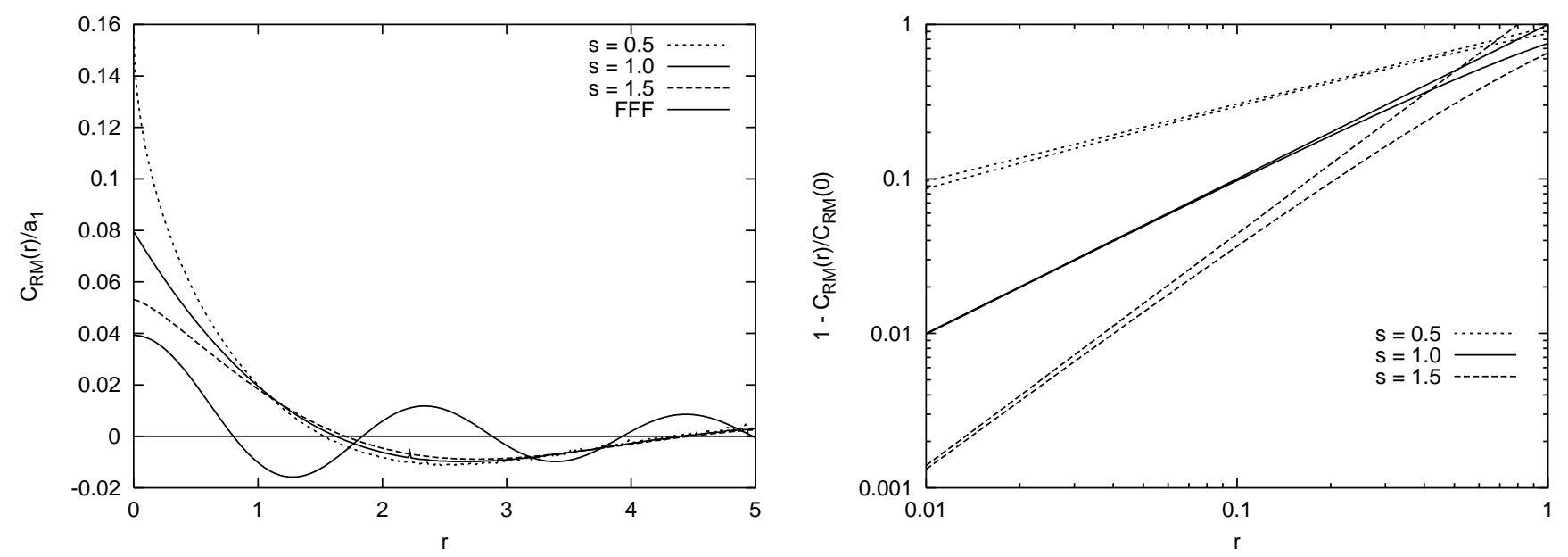

Fig. 1. Left: $C_{\perp}\left(r_{\perp}\right)$ for power-law spectra of magnetic fluctuations $\hat{w}(k)=\hat{w}_{0} k^{-s-2} \mathbf{1}_{\left\{k_{1}<k<k_{2}\right\}}$ within the range $k_{1}=1$ to $k_{2}=10^{4}$ with normalisation $\hat{w}_{0}=1$ and different slopes $s$ as labelled. For comparison, the thin line shows the $R M$ correlation function expected for a singlescale power spectrum, as it can arise for isotropic linear force-free fields $\left(C_{\perp}\left(r_{\perp}\right)=\pi\left\langle B^{2}\right\rangle J_{0}\left(k_{\mathrm{F}} r_{\perp}\right) /\left(2 k_{\mathrm{F}}\right)\right.$ with $k_{\mathrm{F}}=3$, and $\left\langle B^{2}\right\rangle=0.075$; see Sect. 3.4). Right: $1-C_{\perp}\left(r_{\perp}\right) / C_{\perp}(0)$ in logarithmic units for the same power-law spectra. The unbent lines are the asymptotic spectra given by the first two terms in Eq. (44).

Also the correlation lengths can be expressed in terms of $\hat{w}(k)$ :

$\lambda_{B}=\pi \frac{\int_{0}^{\infty} \mathrm{d} k k \hat{w}(k)}{\int_{0}^{\infty} \mathrm{d} k k^{2} \hat{w}(k)}, \quad \lambda_{R M}=2 \frac{\int_{0}^{\infty} \mathrm{d} k \hat{w}(k)}{\int_{0}^{\infty} \mathrm{d} k k \hat{w}(k)}$.

Thus, the $R M$ correlation length has a much larger weight on the large-scale fluctuations than the magnetic correlation length has. Equating these two length-scales, as sometimes done in the literature, is at least questionable in the likely case of a broader turbulence spectrum. In typical situations (e.g. for a broad maximum of the magnetic power spectrum as often found in hydrodynamical turbulence) one expects $\lambda_{B}<\lambda_{R M}$. Since the former is the one which enters the magnetic field estimates by using the measured $R M$-dispersion,

$\left\langle B^{2}\right\rangle=\frac{2 C_{R M}(0)}{a_{1} \lambda_{B}}=\frac{2\left\langle R M^{2}\right\rangle}{a_{0}^{2} n_{\mathrm{e}}^{2} L \lambda_{B}}$, with $L=\frac{V_{[f]}}{A_{\Omega}}$,

using the easily measurable $\lambda_{\mathrm{RM}}$ instead of $\lambda_{B}$ likely underestimates the magnetic field strength.

The isotropic magnetic autocorrelation function can be expressed as

$w(r)=\frac{4 \pi}{(2 \pi)^{3}} \int_{0}^{\infty} \mathrm{d} k k^{2} \hat{w}(k) \frac{\sin (k r)}{k r}$.

Similarly, the $R M$ autocorrelation function can be written as

$C_{\perp}\left(r_{\perp}\right)=\frac{1}{4 \pi} \int_{0}^{\infty} \mathrm{d} k k \hat{w}(k) J_{0}\left(k r_{\perp}\right)$,

where $J_{n}(x)$ is the $n$th Bessel function. In order to analyse the behaviour of the $R M$ autocorrelations close to the origin, it is useful to rewrite the last equation as:

$C_{\perp}\left(r_{\perp}\right)=\int_{0}^{\infty} \mathrm{d} k \frac{k \hat{w}(k)}{4 \pi}-\int_{0}^{\infty} \mathrm{d} k \frac{k \hat{w}(k)}{4 \pi}\left(1-J_{0}\left(k r_{\perp}\right)\right)$.

The first term gives $C_{\perp}(0)$, and the second describes how $C_{\perp}\left(r_{\perp}\right)$ approaches zero for $r_{\perp} \rightarrow \infty$.

\subsection{Power-law power spectra}

In many cases the small-scale magnetic energy spectrum is a power-law, say $\varepsilon_{B}(k)=\varepsilon_{0} k^{-s}$ (e.g. $s=5 / 3$ for Kolmogorovlike turbulence, as expected if the magnetic fields were shaped by a mostly hydrodynamical turbulence) or $w_{B}(k)=\hat{w}_{0} k^{-2-s}$ for $k_{1}<k<k_{2}$ (with $k_{1} \ll k_{2}$ ). For the behaviour of $C_{\perp}\left(r_{\perp}\right)$ on such scales Eq. (43) can be written as

$C_{\perp}\left(r_{\perp}\right)=C_{\perp}(0)-G(s) \hat{w}_{0} r_{\perp}^{s}+R_{[w]}\left(r_{\perp}\right)$,

where

$C_{\perp}(0)=\int_{0}^{\infty} \mathrm{d} k \frac{k \hat{w}(k)}{4 \pi}$,

$G(s)=\frac{\Gamma\left(\frac{2-s}{2}\right)}{2^{s+2} \pi s \Gamma\left(\frac{2+s}{2}\right)}$ for $0<s<2$, and

$R_{[w]}(r)=\frac{1}{4 \pi} \int_{0}^{\infty} \mathrm{d} k k\left(\hat{w}(k)-\hat{w}_{0} k^{-s-2}\right)\left(1-J_{0}(k r)\right)$.

On small scales (more specifically for $k_{1} \ll 1 / r_{\perp} \ll k_{2}$ ) and for well behaved power spectra outside $k_{1} \ll k \ll k_{2}$ the term $R_{[w]}(r)$ is negligibly small. We therefore propose to fit

$C_{R M}\left(r_{\perp}\right) \approx C_{0}-C_{1} r_{\perp}^{s}$

to the inner part of an observationally determined $R M$ correlation function. From this, the turbulence spectral index $s$, $C_{\perp}(0)=C_{0} / a_{1}$, and the power-law normalisation $w_{0}=$ $C_{1} /\left(a_{1} G(s)\right)$ can be inferred. A rough estimate of $k_{1}$ and $k_{2}$, the scales on which the spectrum deviates from the power-law, can also be obtained from finding the $r_{\perp}$-values, where the fit becomes poor. A more accurate determination of these scales can always be done in the Fourier domain (see Eqs. (32), (36) and (37)). We therefore recommend the usage of Eq. (48) more for consistency checks and rapid and rough fit-by-eye diagnostics of the steepness of the magnetic power spectrum rather than for high-precession analysis. 
The shape of the $R M$ correlation function close to the origin allows a direct read-off of the type of magnetic turbulence. A top-down scenario, where most of the energy resides on large scales $(s>1)$ and the smaller scales are populated by a turbulent cascade as in the Kolmogorov-, Kraichnan-, and Goldreich-Sridhar-phenomenologies, leads to a flat cusp at the origin, and a convex shape near to it. A bottom-up magnetic turbulence scenario, where the fields originate on small scales and are enlarged by shear flows or other inverse cascade actions $(s<1)$, leads to a sharp cusp at the origin, and a concave slope next to it. A spectral energy distribution with as much energy on small as on large scales $(s=1)$ leads to a linear cusp at the origin. The behaviour of $C_{\perp}\left(r_{\perp}\right)$ for these three cases is illustrated in Fig. 1.

\subsection{Helical correlations and force-free fields}

Faraday rotation maps do not contain information about the helical part of the autocorrelation tensor. Therefore, additional information is required in order to be able to measure the helical correlations. For example any relation between the helical and non-helical components would be sufficient.

In order to give an example for such additional information, we discuss the case of force-free fields (FFFs). The condition for FFFs reads $\boldsymbol{\nabla} \times \boldsymbol{B}=k_{\mathrm{F}} \boldsymbol{B}$, where $k_{\mathrm{F}}$ can in general be a function of position. For simplicity, we restrict $k_{\mathrm{F}}$ to be spatially constant. Such so-called linear FFFs lead to a very simple structure of the components of $\hat{M}_{i j}(\boldsymbol{k})$ : for $k \neq k_{\mathrm{F}}$ all components vanish, and for $k=k_{\mathrm{F}}$ one gets $\hat{M}_{\mathrm{N}}\left(k_{\mathrm{F}}\right)=-\hat{H}\left(k_{\mathrm{F}}\right)$, leaving the magnetic energy density (or the helicity) as the only remaining free parameter for a given characteristic wavevector $k_{\mathrm{F}}$. FFFs are therefore also called maximally helical fields.

From the fact that for a linear FFF only one spherical shell in wave-vector space is populated with magnetic power, the spatial autocorrelation function is easily obtained as

$w_{\mathrm{F}}(r)=\left\langle B^{2}\right\rangle \sin \left(k_{\mathrm{F}} r\right) /\left(k_{\mathrm{F}} r\right)$

(Subramanian 1999), and the $R M$ autocorrelation as

$C_{\perp}\left(r_{\perp}\right)=\pi\left\langle B^{2}\right\rangle J_{0}\left(k_{\mathrm{F}} r_{\perp}\right) /\left(2 k_{\mathrm{F}}\right)$.

This function is shown on the left side of Fig. 1.

\subsection{Finite window functions}

Here, we discuss the effect of a finite window function on magnetic field estimates, in order to possibly correct for the bias made with the robust weighting scheme introduced in Sect. 2.1. Taking a finite window function $f(\boldsymbol{r})$ into account, Eq. (32) becomes

$\hat{C}_{\perp}^{\mathrm{obs}}\left(\boldsymbol{k}_{\perp}\right)=\frac{1}{2} \int \mathrm{d}^{3} q \hat{w}(q) \frac{q_{\perp}^{2}}{q^{2}} W\left(\boldsymbol{k}_{\perp}-\boldsymbol{q}\right)$,

where we introduced

$W(\boldsymbol{k})=\frac{|\hat{f}(\boldsymbol{k})|^{2}}{(2 \pi)^{3} V_{[f]}}$,

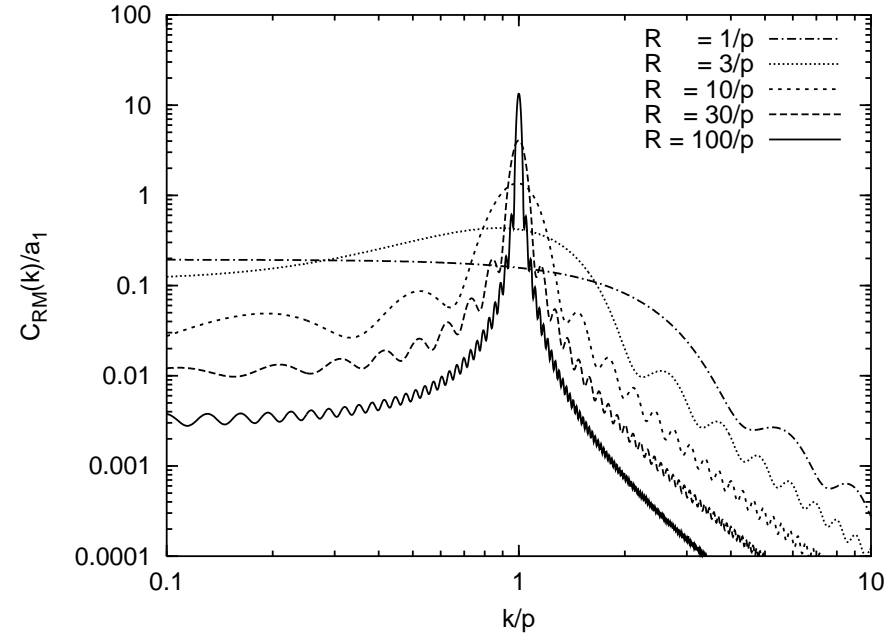

Fig. 2. Response in $\hat{C}_{\perp}\left(k_{\perp}\right)$ to a single-scale magnetic power signal $\hat{w}(k)=\delta(k-p)$ at wavenumber $p$ for different radii $R$ of a cylindrical window function.

and used the identity $\hat{M}_{z z}(\boldsymbol{k})=\frac{1}{2} \hat{w}(k)\left(1-k_{z}^{2} / k^{2}\right)$. Without the term $q_{\perp}^{2} / q^{2}$ the convolution integral in Eq. (51) would describe a redistribution of the magnetic power within Fourier space, which conserves the total magnetic energy. But the term $q_{\perp}^{2} / q^{2}$ leads to some loss of magnetic power.

In any situation in which there is substantial magnetic power on scales comparable or larger than the window size Eq. (51) can be used to estimate the response of the observation to the magnetic power on a given scale $p$ by inserting $\hat{w}(q)=\delta(q-p)$. Ideally, this is then used within a matched-filter analysis or as the response matrix in a maximum-likelihood reconstruction of the underlying power-spectra. The computation of the response matrix relating input power $\hat{w}(q)$ and measured signal $\hat{\boldsymbol{C}}_{\perp}^{\mathrm{obs}}\left(\boldsymbol{k}_{\perp}\right)$ can be cumbersome since in general a 2- or 3-dimensional integral (depending if one uses the delta function) has to be evaluated for each matrix element. Therefore we restrict our discussion here to three highly symmetric, idealised cases, and an approximative treatment of a more realistic configuration, which should give a feeling for the general behaviour.

A cylindrical window: suppose a circular radio source with radius $R$ is seen through a very deep Faraday screen, so that the depth $L_{z}$ can be approximated to be infinite long. The window function $f(\boldsymbol{x})=\mathbf{1}_{\left\{x_{\perp}<R\right\}}$ leads to

$W(\boldsymbol{k})=\frac{J_{1}^{2}\left(k_{\perp} R\right)}{\pi k_{\perp}^{2}} \delta\left(\boldsymbol{k}_{z}\right)$,

where $\delta(k)$ is the Dirac's delta function. Inserting this into Eq. (51) gives

$\hat{C}_{\perp}^{\text {obs }}\left(\boldsymbol{k}_{\perp}\right)=\frac{p}{2 \pi} \int_{0}^{2 \pi} \mathrm{d} \phi \frac{J_{1}^{2}\left(\sqrt{k_{\perp}^{2}+p^{2}-2 p k_{\perp} \cos \phi} R\right)}{k_{\perp}^{2}+p^{2}-2 p k_{\perp} \cos \phi}$,

which is shown in Fig. 2. One can clearly see that with increasing window size the response becomes more and more deltafunction-like.

A sheet-like window seen edge-on: suppose a very elongated radio source is seen through a deep Faraday screen, so 


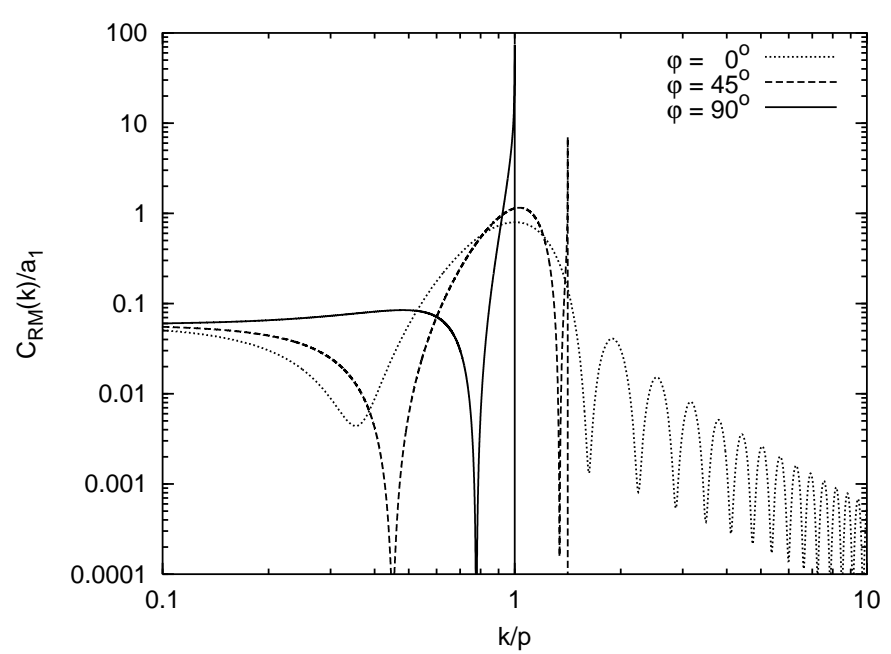

Fig. 3. Response in $\hat{C}_{\perp}\left(k_{\perp}(\cos \phi, \sin \phi)\right)$ to a single-scale magnetic power signal $\hat{w}(k)=\delta(k-p)$ at wavenumber $p$ for a sheet-like window seen edge-on with diameter $L_{x}=10 / p$ for different wavevector orientations $\phi$ with respect to the sheet normal.

that the window function is approximated by $f(\boldsymbol{x})=\mathbf{1}_{\left\{0<x<L_{x}\right\}}$. This gives

$W(\boldsymbol{k})=\Delta_{L_{x}}\left(k_{x}\right) \delta\left(k_{y}\right) \delta\left(k_{z}\right)$,

where we introduced a shortcut for the Fourier transformed 1-d-box-window of size $L$ :

$\Delta_{L}(k)=\frac{L}{2 \pi} \frac{\sin ^{2}(k L / 2)}{(k L / 2)^{2}} \rightarrow \delta(k)$ for $L \rightarrow \infty$.

This leads to a response of

$\hat{C}_{\perp}^{\mathrm{obs}}\left(\boldsymbol{k}_{\perp}\right)=\mathbf{1}_{\left\{k_{y}<p\right\}} \frac{p}{2 t}\left(\Delta_{L_{x}}\left(k_{x}-t\right)+\Delta_{L_{x}}\left(k_{x}+t\right)\right)$,

where we wrote $t=\left(p^{2}-k_{y}^{2}\right)^{1 / 2}$ for brevity. The anisotropic response of this window is much more delta-function like for wavevectors oriented along the sheet axis than oriented perpendicular to it, as can be seen in Fig. 3.

A sheet-like window seen face-on: suppose the window is an infinitely extended homogeneous layer of thickness $L_{z}$ in $z^{-}$ direction, e.g. a magnetised skin layer of a large radio source, so that $f(\boldsymbol{x})=\mathbf{1}_{\left\{0<z<L_{z}\right\}}$, and

$W(\boldsymbol{k})=\Delta_{L_{z}}\left(k_{z}\right) \delta^{2}\left(\boldsymbol{k}_{\perp}\right)$,

where $\delta^{2}\left(\boldsymbol{k}_{\perp}\right)$ is the 2-d Dirac's delta function.

The response function

$\hat{C}_{\perp}^{\mathrm{obs}}\left(\boldsymbol{k}_{\perp}\right)=\mathbf{1}_{\left\{k_{\perp}<p\right\}} \frac{k_{\perp}^{2}}{p \sqrt{p^{2}-k_{\perp}^{2}}} \Delta_{L_{z}}\left(\sqrt{p^{2}-k_{\perp}^{2}}\right)$

is isotropic, and is shown in Fig. 4. In this geometry, power gets scattered out of the $k_{z}=0$ plane due to the finite window size in $z$-direction. This leads to a loss of magnetic power in any measurement, which does not correct for this bias.

The observed magnetic energy density estimated with the help of Eq. (38) can be shown to be related to the real magnetic power spectrum via

$\varepsilon_{B}^{\mathrm{obs}}=\int_{0}^{\infty} \mathrm{d} k \varepsilon_{B}(k) D(k)$,

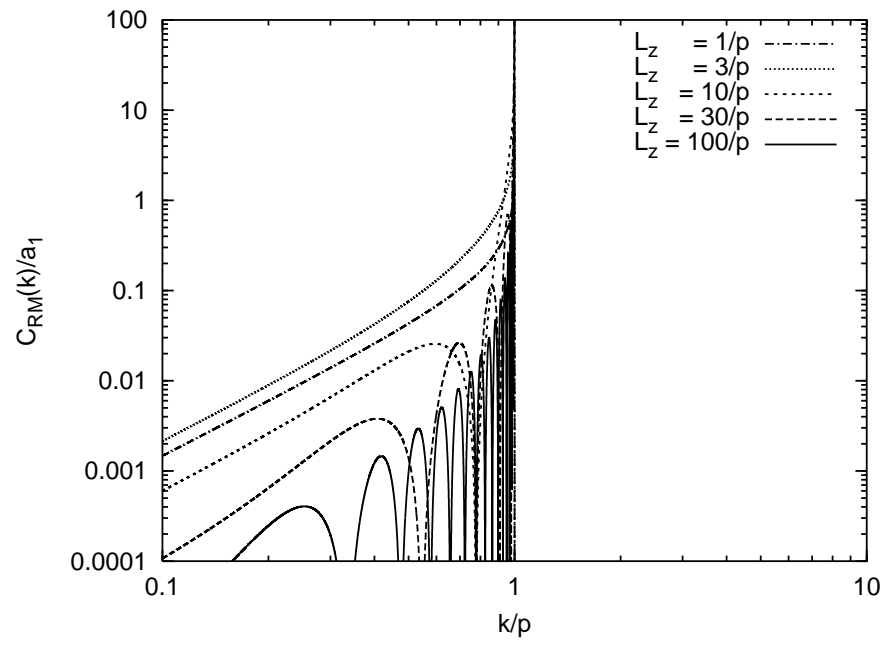

Fig. 4. Response in $\hat{C}_{\perp}\left(k_{\perp}\right)$ to a single-scale magnetic power signal $\hat{w}(k)=\delta(k-p)$ at wavenumber $p$ for a sheet-like window seen face-on for different diameters $L_{z}$.

where the weighting function

$$
\begin{aligned}
D(k) & =\frac{3}{2 k L_{z}}\left[{ }_{1} \mathrm{~F}_{2}\left(\frac{1}{2} ; \frac{1}{2}, 2 ;-\left(\frac{k L_{z}}{2}\right)^{2}\right)-1\right] \\
& \approx \frac{k L_{z}}{\sqrt{(16 / 3)^{2}+\left(k L_{z}\right)^{2}}}= \begin{cases}3 k L_{z} / 16 & ; k L_{z} \ll 1 \\
1 & ; k L_{z} \gg 1\end{cases}
\end{aligned}
$$

describes the relative contribution of different parts of the spectrum. Note that $\varepsilon_{B}(k) D(k)$ is not the observed power spectrum, but only the contribution to the magnetic energy estimate, that ${ }_{a} \mathrm{~F}_{b}$ denotes the hypergeometric function, and that the asymptotic approximation has an overall accuracy of better than $5 \%$. Since $D(k)<1$ everywhere, it is obvious that the derived magnetic fields are underestimated, especially if there is substantial magnetic power on scales comparable and larger than the window size.

In principle, it is possible to correct for any bias, if the window function is reliably known and if the statistical sampling is sufficiently good even on the large scales so that dividing the observed magnetic power spectrum by the weighting function gives sensible results (and not just amplifies the noise).

Approximative treatment of a realistic window: in a realistic situation, often a relatively small sized radio galaxy is seen through a deep Faraday screen. In such a case the depth can again be approximated to be infinite for the purpose of the Fourier-space window:

$W(\boldsymbol{k})=\delta\left(k_{z}\right) W_{\perp}\left(\boldsymbol{k}_{\perp}\right)$,

where we introduced the projected Fourier-window

$W_{\perp}\left(\boldsymbol{k}_{\perp}\right)=\frac{\left|\hat{f}_{\perp}\left(\boldsymbol{k}_{\perp}\right)\right|^{2}}{(2 \pi)^{2} A_{\left[f_{\perp}\right]}}$,

which results from a projected window function:

$f_{\perp}^{2}\left(\boldsymbol{x}_{\perp}\right)=\int \mathrm{d} z f^{2}(\boldsymbol{x}) / L_{z}$ with $A_{\left[f_{\perp}\right]}=\int \mathrm{d}^{2} x_{\perp} f_{\perp}^{2}\left(\boldsymbol{x}_{\perp}\right)$. 
Here, $L_{z}$ is an arbitrary but fixed reference length, e.g. the typical source size $L_{z}=V_{[f]} / A_{\Omega}$. In the case that the observationally measured magnetic power spectrum results from a spherical average over the data

$\hat{w}^{\mathrm{obs}}(k)=\frac{1}{2 \pi} \int_{0}^{2 \pi} \mathrm{d} \phi 2 \hat{C}_{\perp}^{\mathrm{obs}}(k(\cos \phi, \sin \phi))$

the response to a delta function-like magnetic power-spectrum $\hat{w}(q)=\delta(q-p)$ is given by

$$
\begin{aligned}
\hat{w}_{p}^{\mathrm{obs}}\left(k_{\perp}\right) & =\frac{2 p}{\pi} \int \mathrm{d}^{2} q_{\perp} \frac{W_{\perp}\left(\boldsymbol{q}_{\perp}\right) \mathbf{1}_{\left\{\left|k_{\perp}-p\right| \leq q_{\perp} \leq k_{\perp}+p\right\}}}{\sqrt{4 q_{\perp}^{2} p^{2}-\left(q_{\perp}^{2}+p^{2}-k_{\perp}^{2}\right)^{2}}} \\
& =\frac{2 p}{\pi} \int_{\left|k_{\perp}-p\right|}^{k_{\perp}+p} \mathrm{~d} q \frac{q \int_{0}^{2 \pi} \mathrm{d} \phi W_{\perp}(q(\cos \phi, \sin \phi))}{\sqrt{4 q^{2} p^{2}-\left(q^{2}+p^{2}-k_{\perp}^{2}\right)^{2}}} .
\end{aligned}
$$

This approximative response can be easily computed numerically for any model window function. In many cases it should be sufficiently accurate to estimate the effect of a finite observational window on the derived magnetic power spectrum. As a consistency check, we verified that the limit of an infinitely extended radio source, which can be written as $u \rightarrow 0$ in $W_{\perp}\left(\boldsymbol{q}_{\perp}\right)=\mathbf{1}_{\left\{q_{\perp} \leq u\right\}} /\left(\pi u^{2}\right)$, leads to $\hat{w}_{p}^{\mathrm{obs}}(q)=\delta(q-p)$ as it should.

\subsection{Testing isotropy}

Since isotropy of the magnetic field statistics is a crucial ingredient of the proposed analysis, it is important to test if indications of anisotropy are present, and to see how anisotropy can affect the results.

Anisotropy can manifest itself in two different ways: (a) The Fourier space magnetic power distribution can be anisotropic, by being not only a function of $k$ but a full function of $\boldsymbol{k}$, and (b) the magnetic power tensor itself can be anisotropic. Certainly both flavours of anisotropy can be present simultaneously. However, their effects can be well separated, so that we discuss them one by one.

Before doing so, we note that the relation

$\hat{C}_{\perp}\left(\boldsymbol{k}_{\perp}\right)=\hat{M}_{z z}\left(\boldsymbol{k}_{\perp}, 0\right)$

is completely independent on assumptions of isotropy. The $R M$ power spectrum still reveals the $k_{z}=0$ plane of the $z z$-component of the magnetic power tensor. The condition of isotropy had allowed to use the measured information as a representative probe of (a) the full Fourier-space and (b) the other diagonal elements of the magnetic tensor.

(a) Anisotropic power spectrum: Eq. (69) shows that an anisotropic power spectrum can be detected, since it leads very likely to an anisotropic $R M$ power map if the anisotropy is not aligned with the $z$-direction by chance. Since the latter can not be excluded, it is hard to prove isotropy. On the other hand, a perfect alignment of the line-of-sight and the anisotropy axis is not very likely. By studying a number of independent Faraday screens, an anisotropic power spectrum can be ruled out on a statistical basis. Furthermore, by co-adding the signals of several systems, statistical isotropy can be enforced, even if an individual system is anisotropic. However, in order to be able to co-add different observations, the window functions have to be well understood. Especially the scaling of the average magnetic field energy density with location within the Faraday screen and from screen-to-screen should be known. Since this is still poorly known, it is worth to check if indications of anisotropy are present in every dataset itself.

A good way to check for indications of anisotropy is by eye inspection of maps of $\hat{C}_{\perp}\left(\boldsymbol{k}_{\perp}\right)$ or equivalently $C_{\perp}\left(\boldsymbol{r}_{\perp}\right)$ or by comparing profiles which were calculated using different angular slices. A more quantitative estimate of apparent anisotropy can be obtained by the use of multipole moments. Since the dipole moment vanishes due to mirror symmetries in $\hat{C}_{\perp}\left(\boldsymbol{k}_{\perp}\right)$ and $C_{\perp}\left(\boldsymbol{r}_{\perp}\right)$, the first non-trivial multipole is the quadrupole moment:

$Q_{i j}^{(\gamma)}=\int \mathrm{d}^{2} k_{\perp} \hat{C}_{\perp}\left(\boldsymbol{k}_{\perp}\right)\left(2 k_{i} k_{j}-k_{\perp}^{2} \delta_{i j}\right) k_{\perp}^{-\gamma}$

(and similarly for $C_{\perp}\left(r_{\perp}\right)$ ). Here we allowed for a weighting factor $k_{\perp}^{-\gamma}$ in order to balance the contributions from different scales. $Q^{(\gamma)}$ should be compared to the second moment of the (weighted) distribution

$P_{2}^{(\gamma)}=\int \mathrm{d}^{2} k_{\perp} \hat{C}_{\perp}\left(\boldsymbol{k}_{\perp}\right) k_{\perp}^{2-\gamma} \quad\left(\right.$ and similarly for $\left.C_{\perp}\left(r_{\perp}\right)\right)$,

e.g. by calculating the ratio $R^{(\gamma)}=\left(\left|Q_{x x}^{(\gamma)}\right|+\left|Q_{x y}^{(\gamma)}\right|\right) /\left(2 P_{2}^{(\gamma)}\right)$. In an isotropic case this number should be close to zero, in a strongly anisotropic case it can become comparable with one. We suggest to apply this test to the real-space and the Fourier-space data, since in the last case isotropy is mostly tested on large spatial scales, and in the second case on small spatial scale. Attention has to be given to the fact that the realspace quadrupole moment is sensitive (for small $\gamma$ ) to any noncircularity of the window function which can affect large $\boldsymbol{r}_{\perp}$. The Fourier-space quadrupole moment can be affected (for small $\gamma$ ) by any ellipticity of the synthesised beams of the observations since this manifests itself at large $\boldsymbol{k}_{\perp}$. Therefore the integration range for the quadrupole moments and the second moments may be better restricted to intermediate radii in realand in Fourier-space.

(b) Anisotropic tensor: in the anisotropic case, the only constraint on the magnetic autocorrelation tensor is $k_{i} \hat{M}_{i j}(\boldsymbol{k})=$ 0 due to the divergence-freeness of the magnetic fields. This translates for the $z$-components into $k_{x} \hat{M}_{x z}(\boldsymbol{k})+k_{y} \hat{M}_{y z}(\boldsymbol{k})+$ $k_{z} \hat{M}_{z z}(\boldsymbol{k})=0$, which leaves the observable $\hat{M}_{z z}\left(\boldsymbol{k}_{\perp}, 0\right)$ absolutely unconstrained since $k_{z}=0 . \hat{M}_{z z}$ can therefore be an arbitrary function of $\boldsymbol{k}_{\perp}$. However, if it is not circularly symmetric, this can be detected with the methods described above.

In order to have a working example of an anisotropic part $\hat{M}_{i j}^{a}$ of the magnetic tensor we assume that a preferred direction $\boldsymbol{a}(k)$ exists, so that

$\hat{M}_{i j}^{a}(\boldsymbol{k})=\left(a_{i}-\frac{k_{i} a_{l} k_{l}}{k^{2}}\right)\left(a_{j}-\frac{k_{j} a_{l} k_{l}}{k^{2}}\right)$.

This is an intrinsically anisotropic tensor, which fulfils $k_{i} \hat{M}_{i j}^{a}(\boldsymbol{k})=0$. The Faraday-observable component is $\hat{M}_{i j}^{a}\left(\boldsymbol{k}_{\perp}, 0\right)=a_{z}^{2}\left(k_{\perp}\right)$. Its influence on the $R M$ statistics can not be discriminated from an isotropic contribution if $a(k)$ depends only on $|\boldsymbol{k}|$. The assumption of isotropy would therefore 
lead to an incorrect estimate of the field strength, since the measured $z z$-component is assumed to be representative for all components. However, if the signal from a number of similarly anisotropic Faraday screens are co-added the errors compensate statistically, if no correlation of the anisotropic direction and the line-of-sight are present.

Furthermore, since any anisotropy of the magnetic power tensor should have a physical cause, e.g. a large-scale shear flow in the Faraday active medium, an accompanying anisotropic power spectrum is very likely, which can principally be detected by the methods described above (a).

Finally, if anisotropy turns out to be inherently present in Faraday screens, one might replace Eqs. (8) and (28) by a more complex, anisotropic model in order to be able to extract information from individual screens. In that case this work may help as a guideline for such a more elaborate analysis.

\section{Magnetic structures}

\subsection{Autocorrelation}

The possibility exists that the magnetic fields of a Faraday screen consist of several distinct magnetic structures like flux ropes, magnetic tori etc. If the positions and orientations of the structures can be regarded as statistically independent the magnetic autocorrelation function can be written as

$w(r)=\sum_{s} n_{s} W_{s}(r)$,

where all types $s$ of structures present with space density $n_{s}$ are summed up. A structure $s$ with field configuration $\boldsymbol{B}_{s}(\boldsymbol{x})$ has an intrinsic isotropically averaged (unnormalised) autocorrelation function

$W_{s}(r)=\frac{1}{4 \pi} \int \mathrm{d}^{2} \Omega \int \mathrm{d}^{3} x \boldsymbol{B}_{s}(\boldsymbol{x}) \cdot \boldsymbol{B}_{s}(\boldsymbol{x}+\boldsymbol{\Omega} r)$,

where the first integration covers the unit sphere.

For a magnetic structure, which consists of a mostly constant magnetic field $B_{s}$ within the volume $V_{s}$, and negligible field strength elsewhere, the autocorrelation function is asymptotically for small $r$

$W_{s}(r)=B_{s}^{2} V_{s}\left(1-r / l_{s}\right)$,

where $l_{s}$ is a typical length-scale of the structure, roughly given by $l_{s} \sim V_{s} / A_{s}$ with $A_{s}$ the surface area of the structure. If only a single type of structure is present, we get asymptotically

$w(r)=B_{s}^{2} \eta_{B}\left(1-r / l_{s}\right)$,

where $\eta_{B}=n_{s} V_{s}$ is the magnetic volume filling factor.

In order to calculate the $R M$ autocorrelation of such a Faraday screen, we use as a toy model $w(r)$ from Eq. (76) as long as $r<r_{\max }$, and otherwise $w(r)=0$. Equation (17) would then requires $r_{\max }=\frac{4}{3} l_{s}$, but the actual choice is only important for numerical values of constants of proportionality, and not for the qualitative shape of the $R M$ autocorrelation function at the origin. Integrating Eq. (13) leads to an asymptotic expansion of the form

$C_{\perp}\left(r_{\perp}\right)=C_{0}-\left[C_{1}+C_{2} \ln \left(\frac{l_{s}}{r_{\perp}}\right)\right]\left(\frac{r_{\perp}}{l_{s}}\right)^{2}$, which gives a flat central slope, nearly a parabola, but still having a tiny logarithmic cusp. The constants $C_{0}, C_{1}$, and $C_{2}$ depend on the details of the outer slope of $w(r)$, e.g. on the ratio $r_{\max } / l_{s}$, so that their numerical values are model dependent.

We summarise that a Faraday screen built from structural elements with internally constant magnetic fields, and only a single characteristic length-scale leads to a flat central autocorrelation function, with at most a logarithmic cusp of the form given by Eq. (77).

\subsection{Filling factor}

Although there exist characteristic shapes of the $R M$ autocorrelation function $C_{\perp}\left(r_{\perp}\right)$ in the case of a patchy magnetised Faraday screen, as demonstrated in Sect. 4.1 , the presence of such patches cannot be deduced from $C_{\perp}\left(r_{\perp}\right)$ alone. Since the phase information is missing, the special form of the cusp arising from magnetic structures as given by Eq. (77) can not be distinguished from a complete random phase turbulence with steep power-law like spectra with spectral index $s \approx 2$, as can be seen from comparison with Eq. (48).

In order to measure the patchiness of the magnetic field distribution in galaxy clusters Clarke et al. (2001) used the area filling factor $\eta_{R M}$ of the line-of-sight of extended radio sources which do not show any $R M$ due to the Faraday screen. For their sources, they concluded that $\eta_{R M}>95 \%$.

If the magnetic fields are in flux-rope like structures, with typical length $l_{\|}$and diameter $l_{\perp}$, the cross section of a flux-rope to be intersected by a line-of-sight is of the order $l_{\|} l_{\perp}$. Their volume filling factor is $\eta_{B} \approx l_{\|} l_{\perp}^{2} n_{\text {rope }}$. If their locations can be regarded as being uncorrelated, the number $K$ of flux ropes intersected by a line-of-sight of length $L_{\mathrm{los}}$ is Poisson-distributed: $P(K)=\Lambda^{K} \exp (-\Lambda) /(K !)$ with $\Lambda \approx \eta_{B} L_{\mathrm{los}} / l_{\|}$. From that it follows by inserting $K=0$ that

$\eta_{B} \approx \frac{l_{\|}}{L_{\mathrm{los}}} \ln \left(\frac{1}{1-\eta_{R M}}\right)$.

For filaments of length $l_{\|} \approx 10 \mathrm{kpc}$ and lines-of-sight of $L_{\mathrm{los}} \approx$ $500 \mathrm{kpc}$ only a small subvolume $\eta_{B}>0.05$ of the clusters actually needs to be magnetised in order to give the large area filling factor found by Clarke et al. (2001).

Another constraint for the magnetic filling factor can be obtained from energetic arguments. The magnetic field energy density in magnetised regions can be expected to be below the environmental thermal energy density $\varepsilon_{\text {th }}$, since otherwise a magnetic structure would expand until it reaches pressure equilibrium. Since the autocorrelation analysis of $R M$ maps is able to provide the volume averaged magnetic field energy density $\left\langle\varepsilon_{B}\right\rangle$, the magnetic volume filling factor can be constrained to be

$\eta_{B}>\frac{\left\langle\varepsilon_{B}\right\rangle}{\varepsilon_{\mathrm{th}}}=0.8 \times 10^{-2} \frac{\left\langle B^{2}\right\rangle}{\mu \mathrm{G}^{2}}\left[\frac{n_{\mathrm{e}}}{10^{-3} \mathrm{~cm}^{-3}} \frac{k T}{\mathrm{keV}}\right]^{-1}$.

In cases of relatively strong average magnetic energy densities, as e.g. in cooling flow clusters, or in case of physical arguments requiring a much lower than equipartition field strength, this can give a tight constraint on the volume filling factor. 


\section{Observational artefacts}

\subsection{Beam smearing}

The finite size $l_{\text {beam }}$ of a synthesised beam of a radio interferometer should smear out $R M$ structures below the beam size, and therefore can lead to a smooth behaviour of the measured $R M$ autocorrelation function at the origin, even if the true autocorrelation function has a cusp there. Substantial changes of the $R M$ on the scale of the beam can lead to beam depolarisation, due to the differentially rotated polarisation vectors within the beam area (Conway \& Strom 1985; Laing 1988; Garrington et al. 1988). Since beam depolarisation is in principal detectable by its frequency dependence, the presence of sub-beam structure can be noticed, even if not resolved (Tribble 1991; Melrose \& Macquart 1998). The magnetic power spectrum derived from a beam smeared $R M$ map should cut-off at large $k \sim \pi / l_{\text {beam }}$.

\subsection{Noise}

Instrumental noise can be correlated on several scales, since radio interferometers sample the sky in Fourier space, where each antenna pair baseline measures a different $k_{\perp}$-vector. It is difficult to understand to which extend noise on a telescope antenna baseline pair will produce correlated noise in the $R M$ map, since several independent polarisation maps at different frequencies are combined in the map making process. We therefore discuss only the case of spatially uncorrelated noise, as it may result from a pixel-by-pixel $R M$ fitting routine. This adds to the $R M$ autocorrelation function

$C_{\text {noise }}^{\text {obs }}\left(\boldsymbol{x}_{\perp}, \boldsymbol{r}_{\perp}\right)=\sigma_{R M, \text { noise }}^{2}\left(\boldsymbol{x}_{\perp}\right) \delta^{2}\left(\boldsymbol{r}_{\perp}\right)$.

In Fourier space, this leads to a constant error for $\hat{w}(k)$

$\hat{w}_{\text {noise }}(k)=2\left\langle\sigma_{R M, \text { noise }}^{2}\right\rangle$

and therefore to an artificial component in the magnetic power spectrum $\varepsilon_{B}^{\mathrm{obs}}(k)$ increasing by $k^{2}$.

If it turns out that for an $R M$ map with an inhomogeneous noise map (if provided by an $R M$ map construction software) the noise affects the small-scale power spectrum too severely, one can try to reduce this by down-weighting noisy regions with a suitable choice of the data weighting function $h\left(\boldsymbol{x}_{\perp}\right)$ which was introduced in Sect. 2.1 for this purpose.

\subsection{RM steps due to the $n \pi$-ambiguity}

An $R M$ map is often derived by fitting the wavelength-square behaviour of the measured polarisation angles. Since the polarisation angle is only determined up to an ambiguity of $n \pi$ (where $n$ is an integer), there is the risk of getting a fitted $R M$ value which is off by $m \Delta R M$ from the true one. $m$ is an integer, and $\Delta R M=\pi\left(\lambda_{\min }^{2}-\lambda_{\max }^{2}\right)^{-1}$ is a constant depending on the used wavelength range from $\lambda_{\min }$ to $\lambda_{\max }$.

This can lead to artifical jumps in RM maps, which will affect the $R M$ autocorrelation function and therefore any derived magnetic power spectrum. In order to get a feeling for this we model the possible error by an additional component in the derived $R M$ map:

$R M^{\mathrm{amb}}\left(\boldsymbol{x}_{\perp}\right)=\sum_{i} m_{i} \Delta R M \mathbf{1}_{\left\{\boldsymbol{x}_{\perp} \in \Omega_{i}\right\}}$

where $\Omega_{i}$ is the area of the $i$ th $R M$ patch, and $m_{i}$ is an integer, mostly +1 or -1 . Assuming that different patches are uncorrelated, the measured $R M$ autocorrelation function is changed by an additional component, which should be asymptotically for small $r_{\perp}$

$C_{R M}^{\mathrm{amb}}\left(r_{\perp}\right)=\Delta R M^{2} \eta_{\mathrm{amb}}\left(1-\frac{r_{\perp}}{l_{\mathrm{amb}}}\right)$,

where $\eta_{\mathrm{amb}}$ is the area filling factor of the ambiguity patches in the $R M$ map, and $l_{\mathrm{amb}}$ a typical patch diameter.

Comparing this with Eq. (48) shows that the artificial power induced by the $n \pi$-ambiguity mimics a turbulence energy spectrum with slope $s=1$, which would have equal power on all scales. A steep magnetic power spectrum can therefore possibly be masked by such artefacts.

Fortunately, for a given observation the value of $\triangle R M$ is known and one can search an $R M$ map for the occurrence of steps by $\triangle R M$ over a short distance (not necessarily one pixel) in order to detect such artefacts.

\section{Conclusions}

We have investigated the statistics of Faraday rotation maps on the level of the autocorrelation function and the power spectrum. We proposed ways to analyse extended Faraday maps in order to reconstruct the magnetic autocorrelation tensor (Eqs. (6) and (34)) from which quantities like the average field strength, the magnetic energy spectrum, and their autocorrelation length can be obtained (Eq. (20)). We showed that under the assumption of isotropy of the observed magnetic field ensemble the symmetric part of the magnetic autocorrelation tensor (Eqs. (8) and (28)) can be reconstructed. This makes use of the condition $\boldsymbol{\nabla} \cdot \boldsymbol{B}=0$ and the additional assumption (which can be tested a-posteriori) that the gradient scale of the electron density (e.g. the core radius of a galaxy cluster) is much larger than the typical field length-scale. The anti-symmetric or helical part of the magnetic correlation tensor can only be measured if additional information is available, e.g. in the case of force-free fields (Sect. 3.4).

The assumption of isotropy of the magnetic field statistics should be justified in cases where a sufficiently large volume of the screen is probed. In principle, it can also be tested by searching for non-circular distortions of the 2-dimensional autocorrelation function (Sect. 3.6).

A further test for statistical isotropy and sufficient sampling of the field fluctuations is the fact that if these conditions are given in a finite Faraday screen (which cannot maintain infinitely long correlations) the rotation measure $(R M)$ autocorrelation area (Eq. (18)) has to vanish. This means that there is a balance between the positively and negatively valued areas of the autocorrelation function. In practice, one would require it to be much smaller than the $R M$ autocorrelation length squared. We note that e.g. the popular magnetic cell-model, in which 
cells are filled by from cell-to-cell independently oriented and internally homogeneous magnetic fields, does not have these properties, since it violates the required $\boldsymbol{\nabla} \cdot \boldsymbol{B}=0$ condition.

Our approach is meant to be applied directly to real data. Effects of incomplete information, due to the limited extent of polarised radio sources, are properly treated in form of a window function (Eqs. (4), (5), and (51)). This window function contains additional information on the screen geometry, and allows for proper bookkeeping of data weighting, in case of noisy data being analysed. Since the window function also requires some working hypothesis about the average magnetic energy density profile of the Faraday screen, ways to test it a posteriori are sketched (Sect. 2.4).

The most efficient way to analyse Faraday rotation maps leads through Fourier space (Sect. 3). The Fourier transformation of a map gives direct insight into the magnetic energy spectrum (Eqs. (35) and (37)), which fully specifies the magnetic autocorrelation function (Eq. (32)). Many quantities of interest can be obtained from it, such as the average field strength (Eq. (38)), the correlation lengths (Eq. (39)), and the bias resulting from the used window function (Sect. 3.5).

The Fourier domain formulation gives also important insight into the real space behaviour of the $R M$ autocorrelation function (Sect. 3.3): a power-law magnetic energy spectrum leads to a cusp at the origin of this function, where the shape of the cusp is determined by the power law index $s$. A steep spectrum $s>1$ (as e.g. expected for turbulent cascades) leads to a flat cusp, whereas a flat spectrum $s<1$ gives a pronounced sharp cusp. The limiting case $s=1$ with equal power on all scales leads to a linear (decreasing) behaviour of the $R M$ autocorrelation function close to the origin.

Such cusps of the $R M$ autocorrelation (or power-law spectra in Fourier space) can be signatures of turbulent cascades, but they can also occur in other situations. We demonstrated that a Faraday screen which is composed of finite magnetic structures of roughly constant field strength lead to a flat cusp, too (Sect. 4.1). We show ways to constrain the magnetic volume filling factor for such magnetic field models (Sect. 4.2). Observational artefacts, like noise or jumps in the measured $R M$ values due to the so called $n \pi$-ambiguity, are able to produce sharp cusps (Sect. 5). We therefore stress the importance to check maps for such distortions and describe ways to do this.

A very important result of this work is that the magnetic autocorrelation length $\lambda_{B}$ (Eq. (20)) is in general not identical to the autocorrelation length of the $R M$ fluctuations $\lambda_{R M}$ (Eq. (21)). $\lambda_{R M}$ is much more strongly weighted towards the large-scale part of the magnetic power spectrum than $\lambda_{B}$ (Eq. (39)). In typical astrophysical situations a broad spectral energy distribution can be expected so that $\lambda_{B}$ will be much smaller than $\lambda_{R M}$. Since $\lambda_{B}$ enters the classical formulae to estimate magnetic field strength from Faraday measurements $\left\langle B^{2}\right\rangle \propto\left\langle R M^{2}\right\rangle / \lambda_{B}$ (see Eq. (40)), but $\lambda_{R M}$ is sometimes used instead, we expect that several published magnetic field estimates from Faraday rotation maps are actually underestimates.

We hope that our work aids and stimulates further observational and theoretical work on the exciting field of Faraday rotation measurements of cosmic magnetic fields in order to give us deeper insight in their fascinating origins and roles in the Universe.

Acknowledgements. We thank Kandaswamy Subramanian for many discussions and important suggestions. We acknowledge further stimulating discussions on $R M$ maps with Matthias Bartelmann, Tracy E. Clarke, Klaus Dolag, Luigina Feretti, Gabriele Giovannini, Federica Govoni, Philipp P. Kronberg, and Robert Laing. We thank Matthias Bartelmann and Tracy Clarke for comments on the manuscript. TAE thanks for the hospitality at the Istituto di Radioastronomia at CRN in Bologna in April 2000, where several of the discussions took place. This work was done in the framework of the EC Research and Training Network The Physics of the Intergalactic Medium.

\section{References}

Beck, R. 2001, Space Sci. Rev., 99, 243

Beck, R., Brandenburg, A., Moss, D., Shukurov, A., \& Sokoloff, D. 1996, ARA\&A, 34, 155

Bicknell, G. V., Cameron, R. A., \& Gingold, R. A. 1990, ApJ, 357, 373

Carilli, C. L., \& Taylor, G. B. 2002, ARA\&A, 40, 319

Cho, J., Lazarian, A., \& Vishniac, E. T. 2002, ApJ, 564, 291

Clarke, T. E., Kronberg, P. P., \& Böhringer, H. 2001, ApJ, 547, L111

Conway, R. G., \& Strom, R. G. 1985, A\&A, 146, 392

Dolag, K., Bartelmann, M., \& Lesch, H. 1999, A\&A, 348, 351

Dolag, K., Bartelmann, M., \& Lesch, H. 2002, A\&A, 387, 383

Dolag, K., Schindler, S., Govoni, F., \& Feretti, L. 2001, A\&A, 378, 777

Dreher, J. W., Carilli, C. L., \& Perley, R. A. 1987, ApJ, 316, 611

Eilek, J. A., \& Owen, F. N. 2002, ApJ, 567, 202

Enßlin, T. A., \& Biermann, P. L. 1998, A\&A, 330, 90

Feretti, L. 1999, in Diffuse Thermal and Relativistic Plasma in Galaxy Clusters, ed. P. S. H. Böhringer, \& L. Feretti, MPE-report, 271, 3

Garrington, S. T., Leahy, J. P., Conway, R. G., \& Laing, R. A. 1988, Nature, 331, 147

Goldreich, P., \& Sridhar, S. 1997, ApJ, 485, 680

Govoni, F., Feretti, L., Murgia, M., et al. 2002, in Matter and Energy in Clusters of Galaxies, ed. S. Bowyer, \& C.-Y. Hwang (Taiwan: Chung-Li) [astro-ph/0211292]

Grasso, D., \& Rubinstein, H. R. 2001, Phys. Rep., 348, 163

Kolatt, T. 1998, ApJ, 495, 564

Kosowsky, A., \& Loeb, A. 1996, ApJ, 469, 1

Kronberg, P. P. 1994, Rep. Prog. Phys., 57, 325

Kulsrud, R. M. 1999, ARA\&A, 37, 37

Laing, R. A. 1988, Nature, 331, 149

Maron, J., \& Goldreich, P. 2001, ApJ, 554, 1175

Melrose, D. B., \& Macquart, J.-P. 1998, ApJ, 505, 921

Ohno, H., Takada, M., Dolag, K., Bartelmann, M., \& Sugiyama, N. 2002, ApJ, 584, 599

Oren, A. L., \& Wolfe, A. M. 1995, ApJ, 445, 624

Rees, M. J. 1987, QJRAS, 28, 197

Sridhar, S., \& Goldreich, P. 1994, ApJ, 432, 612

Subramanian, K. 1999, Phys. Rev. Lett., 83, 2957

Taylor, G. B., Govoni, F., Allen, S. W., \& Fabian, A. C. 2001, MNRAS, 326, 2

Taylor, G. B., \& Perley, R. A. 1993, ApJ, 416, 554

Tribble, P. C. 1991, MNRAS, 250, 726

Widrow, L. M. 2002, Rev. Modern Phys., 74, 775

Wielebinski, R., \& Krause, F. 1993, A\&ARv, 4, 449

Willson, M. A. G. 1970, MNRAS, 151, 1 